\title{
The 5-HTTLPR Polymorphism Affects Network-Based Functional Connectivity in the Visual-Limbic System in Healthy Adults
}

\author{
Hengyi Cao', I, Anais Harneit', I, Henrik Walter 2,II, Susanne Erk², Urs Braun', Carolin Moessnang', \\ Lena S Geiger', Zhenxiang Zang', Sebastian Mohnke², Andreas Heinz ${ }^{2}$, Nina Romanczuk-Seiferth², \\ Thomas Mühleisen ${ }^{3,4}$, Manuel Mattheisen ${ }^{5,6}$, Stephanie H Witt ${ }^{7}$, Sven Cichon ${ }^{3,4,8,9}$, Markus M Nöthen ${ }^{8,10}$, \\ Marcella Rietschel ${ }^{7}$, Andreas Meyer-Lindenberg ${ }^{1,11}$ and Heike Tost ${ }^{*, 1,11}$ \\ 'Department of Psychiatry and Psychotherapy, Central Institute of Mental Health, Medical Faculty Mannheim, University of Heidelberg, \\ Mannheim, Germany; ' Department of Psychiatry and Psychotherapy, Universitätsmedizin Charité, Berlin, Germany; ${ }^{3}$ Institute of Neuroscience and \\ Medicine (INM-I), Research Centre Jülich, Jülich, Germany; ${ }^{4}$ Department of Biomedicine, University of Basel, Basel, Switzerland; ${ }^{5}$ Department of \\ Genomic Mathematics, University of Bonn, Bonn, Germany; ${ }^{6}$ Department of Biomedicine, University of Aarhus, Aarhus, Denmark; ${ }^{7}$ Department of \\ Genetic Epidemiology in Psychiatry, Central Institute of Mental Health, Medical Faculty Mannheim, University of Heidelberg, Mannheim, Germany; \\ ${ }^{8}$ Department of Genomics, Life \& Brain Center, University of Bonn, Bonn, Germany; 'Division of Medical Genetics, University of Basel, Basel, \\ Switzerland; ${ }^{10}$ Institute of Human Genetics, University of Bonn School of Medicine \& University Hospital of Bonn, Bonn, Germany
}

\begin{abstract}
The serotonin transporter-linked polymorphic region 5-HTTLPR is a key genetic regulator of 5-HTT expression in the human brain where the short allele $S$ has been implicated in emotion dysregulation. However, the neural mechanism underlying the association between this variant and emotion processing is still unclear. Earlier studies suggested an effect of 5-HTTLPR on amygdala activation during emotional face processing. However, this association has been questioned in recent studies employing larger sample sizes and meta-analyses. Here, we examined a sample of 223 healthy subjects with a well-established $\mathrm{fMRI}$ emotional face processing task to (I) re-evaluate the association between 5-HTTLPR and amygdala activation, (2) explore potential network-based functional connectivity phenotypes for associations with 5-HTTLPR, and (3) probe the reliability, behavioral significance and potential structural confounds of the identified network phenotype. Our results revealed no significant effect of 5-HTTLPR on amygdala activation $(P>0.79)$. However, the number of $S$ alleles was significantly correlated with functional connectivity of a visual-limbic subnetwork $\left(P_{F W E}=0.03\right)$. The subnetwork cluster included brain regions that are pivotal to emotion regulation such as the hippocampus, orbitofrontal cortex, anterior cingulate gyrus, fusiform gyrus, and subcortex. Notably, individuals with lower subnetwork connectivity had significantly higher emotion suppression scores $(P=0.0 \mathrm{I})$. Further, the connectivity metrics were test-retest reliable and independent from subnetwork gray matter volume and white matter anisotropy. Our data provide evidence for a functional network-based phenotype linking genetic variation in 5-HTTLPR to emotion regulation, and suggest that further critical evaluations of the association between 5-HTTLPR and amygdala activation are warranted. Neuropsychopharmacology (20I8) 43, 406-4I4; doi:I0.1038/npp.2017.121; published online 5 July 2017
\end{abstract}

\section{INTRODUCTION}

The serotonin transporter (5-HTT) is a key regulator for emotion regulation (Hariri and Holmes, 2006). The expression of 5-HTT is primarily modulated by variation in the serotonin transporter-linked polymorphic region (5-HTTLPR), a 43-base-pair insertion/deletion polymorphism in the 5 ' regulatory promoter region of the 5-HTT gene SLC6A4,

* Correspondence: Dr H Tost, Central Institute of Mental Health, Medical Faculty Mannheim, University of Heidelberg, Square J5, Mannheim 68159, Germany, Tel: +49621 17036510 ,

E-mail: heike.tost@zi-mannheim.de

I'These authors contributed equally to this work.

Received 5 March 2017; revised 29 April 2017; accepted 16 May 2017; accepted article preview online 7 June 2017 which results in a short (S) and long (L) allele of the gene (Canli and Lesch, 2007; Hariri and Holmes, 2006). The S allele, relative to the $\mathrm{L}$ allele, is associated with attenuated transcriptional activity of SLC6A4 and lower 5-HTT mRNA expression, which increases vulnerability for emotion dysregulation, anxiety and depression (Canli and Lesch, 2007; Caspi et al, 2003; Hariri and Holmes, 2006; Lesch et al, 1996). Transcriptional activity of the $\mathrm{L}$ allele is further influenced by a common single-nucleotide polymorphism (SNP) rs $25531 \mathrm{~A} / \mathrm{G}$, where the $\mathrm{G}$ allele renders the $\mathrm{L}$ allele functionally similar to the $S$ allele (ie $\mathrm{L}+\mathrm{G}$ is equivalent to the low-expressing S (Hu et al, 2005)).

The neural mechanisms underlying the effects of 5-HTTLPR genotype in humans are still unclear. Early studies with smaller sample sizes showed significantly 
increased amygdala response to negative emotional stimuli in $S$ allele carriers compared with LL homozygotes (Hariri et al, 2005; Hariri et al, 2002; Heinz et al, 2005). However, this finding has not been replicated in more recent studies with larger sample sizes (Bastiaansen et al, 2014; Viviani et al, 2010). Moreover, two recent meta-analyses failed to find a convincing association between genotype and amygdala activation (Bastiaansen et al, 2014; Murphy et al, 2013), which challenged the view that amygdala response is a robust neural mechanism related to the polymorphism.

These conflicting results may relate to at least two factors. First, given the small effect size of common genetic variants, earlier studies with small sample sizes may be more vulnerable to noise and false-positive findings (Bastiaansen et al, 2014; Murphy et al, 2013). Second, as reported previously (Johnstone et al, 2005; Plichta et al, 2012), amygdala activation during emotion processing per se is a rather unreliable phenotype. Our prior work has shown a superior reliability and sensitivity of network-based neuroimaging measures in the study of functional phenotypes (Cao et al, 2016; Cao et al, 2014), suggesting the potential utility of network-based methods for the study of 5-HTTLPR genetic variation.

Apart from the amygdala, emotion processing in humans entails the close cooperation of multiple other regions including the fusiform gyrus, hippocampus, striatum, thalamus, anterior cingulate cortex, insula, and orbitofrontal cortex (Etkin et al, 2015; Phillips et al, 2003). These regions form a functional network that is pivotal for emotion identification and regulation (Etkin et al, 2015; Phillips et al, 2003). Prior work has shown that the connectivity of this emotional network is heritable (Budisavljevic et al, 2016) and has been implicated in the genetic risk for mental disorders including schizophrenia (Cao et al, 2016) and bipolar disorder (Dima et al, 2016). Further, significant alterations have been shown in serotonin signaling in 5-HTT knock-out mice in multiple regions of this network (Fabre et al, 2000; Mathews et al, 2004; Rioux et al, 1999). Human research also demonstrated that the $\mathrm{S}$ allele is associated with altered 5HTT concentrations and serotonin receptor binding potentials in the striatum (Praschak-Rieder et al, 2007), hippocampus, anterior cingulate gyrus, orbitofrontal cortex, and lateral temporal lobe (David et al, 2005). These findings suggest that 5-HTTLPR may impact the connectivity of multiple regions in the emotional processing network in humans.

In this study, we aimed to identify potential connectomic phenotypes related to 5-HTTLPR genetic variation in healthy participants using functional magnetic resonance imaging (fMRI) and a well-established emotional face-matching task (Hariri et al, 2002). Specifically, we aimed to (1) follow-up prior conflicting reports on the effects of this genotype on amygdala activation, (2) search for novel functional phenotypes linked to 5-HTTLPR genotype at the brain network

Table I Sample Characteristics Stratified by 5-HTTLPR Genotype

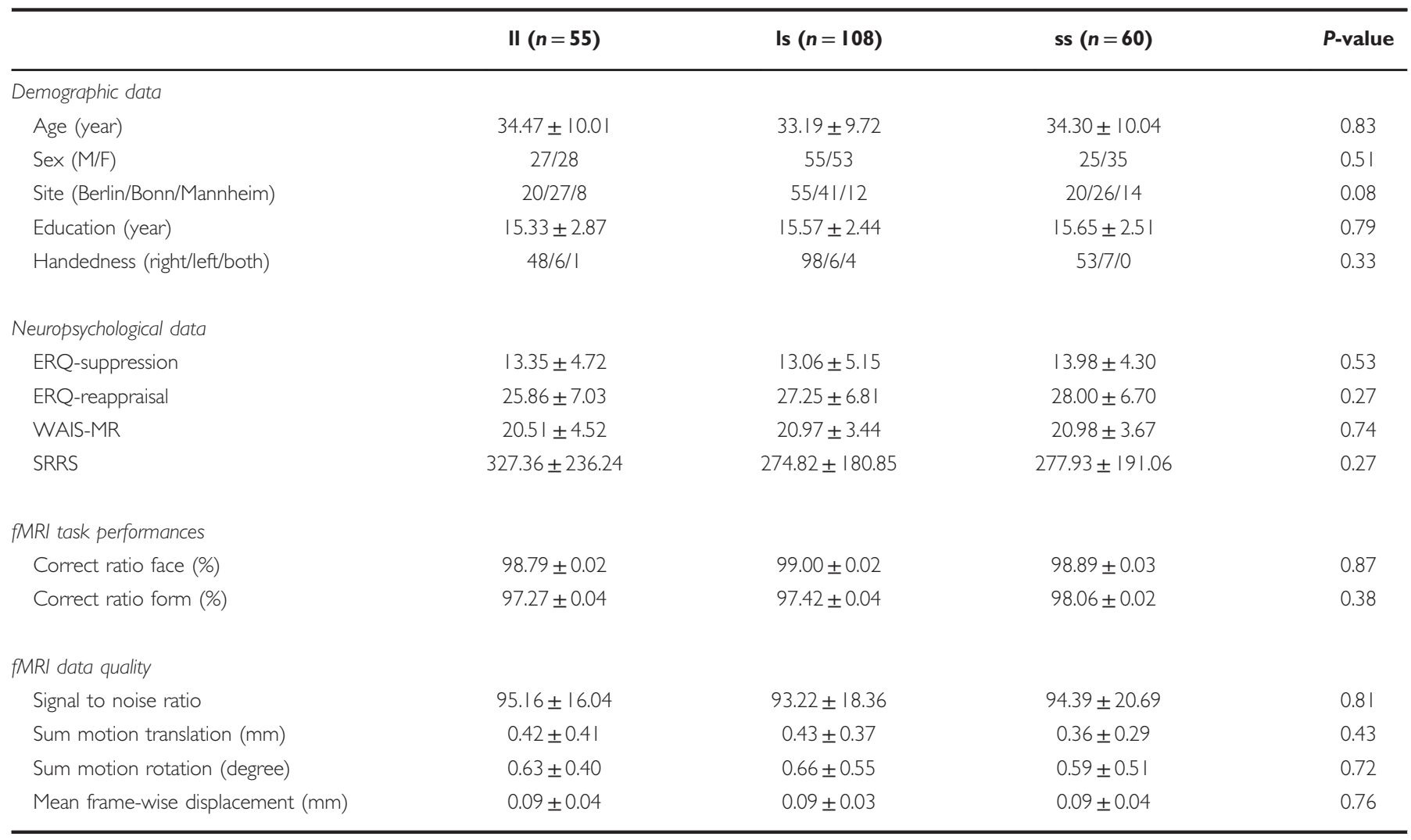

Abbreviations: ERQ: Emotion Regulation Questionnaire; SRRS: Social Readjustment Rating Scale; WAIS-MR: Matrix Reasoning Subscale of Wechsler Adult Intelligence Scale.

Note that ERQ subscale scores were calculated as the sum of contributing item scores, and SRRS were derived from the assessment of life events in the last 2 years. Categorical variables are reported as numbers of cases, continuous variables are reported as mean and SD. 
level, and (3) investigate the utility of the identified networkbased phenotype by probing its test-retest reliability, associations with emotion regulation and potential structural basis.

\section{MATERIALS AND METHODS}

\section{Participants}

A total of 223 healthy subjects with available data on 5-HTTLPR, functional and structural imaging participated in this study (mean age 33.35 \pm 9.92 years, 107 males). The participants were recruited from the communities at three sites in Germany (Mannheim, Bonn, Berlin). The exclusion criteria are given in the Supplementary Materials. All subjects provided written informed consent for protocols approved by the institutional ethical review boards of the Universities of Heidelberg, Bonn and Berlin.

\section{Genotyping}

Details on the genotyping procedures are provided in the Supplementary Materials. In brief, subjects were classified into three groups based on the combination of the L/S and rs25531 A/G polymorphisms: $l l$ group (including $\mathrm{L}_{\mathrm{A}} / \mathrm{L}_{\mathrm{A}}, 55$ subjects), $l s$ group (including $\mathrm{L}_{\mathrm{A}} / \mathrm{L}_{\mathrm{G}}$ and $\mathrm{L}_{\mathrm{A}} / \mathrm{S}, 108$ subjects), and ss group (including $\mathrm{L}_{\mathrm{G}} / \mathrm{L}_{\mathrm{G}}, \mathrm{L}_{\mathrm{G}} / \mathrm{S}$ and $\mathrm{S} / \mathrm{S}, 60$ subjects). The three genotype groups did not show significant differences in demographic, psychological and fMRI performance data (all $P$-values $>0.08$, Table 1 ).

\section{MRI Modalities and Task Paradigm}

Multimodal magnetic resonance imaging (MRI) data were collected from all participants. During the fMRI scan, participants completed a well-established emotional facematching task as previously described (Hariri et al, 2002). In brief, the face-matching task is an implicit emotional processing paradigm consisting of two conditions: an emotional face condition (matching faces) and a control condition (matching forms). In the emotional face condition, subjects were presented with trios of faces depicting fearful or angry expressions and were instructed to match the two facial displays belonging to the same individual. In the control condition, participants were presented with trios of geometric shapes (circles, vertical and horizontal ellipses) and were asked to match the two identical shapes. In addition, we acquired high-resolution T1-weighted images for each individual and diffusion tensor images (DTI) for part of the subjects (141 out of 223). These data allow us to explore potential brain network changes at both the functional and structural level.

\section{Data Acquisition}

See Supplementary Materials for details on data acquisition.

\section{Data Quality Control}

See Supplementary Materials for details on image quality control procedures.

\section{Amygdala Activation Analysis}

We first attempted to replicate the association between 5-HTTLPR and amygdala activation findings reported in previous studies (Hariri et al, 2005; Hariri et al, 2002). Here, we closely followed the methods detailed in these studies and used standard procedures in Statistical Parametric Mapping (SPM8, http://www.fil.ion.ucl.ac.uk/spm/software/spm8/). In brief, functional images were realigned to the first image of the scan, slice time corrected, normalized to the Montreal Neurological Institute brain template with resampling to $3 \times 3 \times 3 \mathrm{~mm}^{3}$ voxels, and spatially smoothed with a $9 \mathrm{~mm}$ full-width at half-maximum Gaussian kernel. The preprocessed images were then subjected to first-level general linear model estimation, where data were high-pass filtered (cutoff $128 \mathrm{~s}$ ) and individual maps of the 'face-matching $>$ formmatching' contrast were computed. The contrast images were used for a second-level random effects analysis. Here, we employed two different models: a linear regression model with genotypes $l l, l s$, and ss encoded as 0,1 and 2, and an analysis of covariance (ANCOVA) model where subjects with risk allele $s$ were compared with those with $l l$ ( $l l v s s$, ie, the same model as used in Hariri et al (Hariri et al, 2002)). In both models, age, sex, and site were included as nuisance covariates. As in prior work, results were reported after family-wise error (FWE) correction across the mask of bilateral amygdala from the Automated Anatomical Labeling atlas (Tzourio-Mazoyer et al, 2002).

\section{Brain Functional Network Analysis}

The functional network analysis followed our previously published procedures (Cao et al, 2016; Cao et al, 2014). Here, mean time series for each of the 270 nodes detailed in Power et al (2011) were extracted from the preprocessed images. Notably, the original Power atlas only contained 264 nodes without bilateral hippocampus, bilateral amygdala, and bilateral ventral striatum. To achieve whole-brain coverage, we included these nodes based on previously published coordinates from meta-analyses (Liu et al, 2011; Sabatinelli et al, 2011; Spreng et al, 2009), thereby increasing the total number of nodes to 270 (one node per region and hemisphere). The time series were then corrected for mean effects of task conditions, white matter and cerebrospinal fluid signals, and six head motion parameters, and were high-pass filtered (cutoff $128 \mathrm{~s}$ ). Afterwards, we computed pairwise Pearson correlation coefficients between the processed time series of each node, resulting in a $270 \times 270$ twodimensional connectivity matrix for each subject.

Following the procedures in our prior study (Cao et al, 2016), we used network-based statistic (NBS) to identify clusters of functional links that were linearly correlated with the number of risk allele $s$. NBS is a method to effectively control cluster-level FWE for link-wise matrical comparisons, which provides a larger power than mass-univariate tests on independent links (Zalesky et al, 2010). Here, an initial linear model for the three genotypes $l l, l s$, and $s s$ (encoded as 0,1 , and 2) was applied to each of the $N(N-1) /$ $2=36315(N=270)$ links in the connectivity matrices, with age, sex, and site as covariates of no interest. This produced a $P$-value matrix representing the probability of accepting the null hypothesis for each link. All links with $P$-values $\leqslant 0.0001$ 
were then thresholded into a set of suprathreshold links, and connected subnetwork clusters were subsequently identified from the set. The significance of the identified clusters was tested by 5000 permutations, where each subject was randomly reassigned into a genotype group during each permutation and the maximal extent of the identified cluster was recalculated. The corrected $P$-value for the identified cluster was determined by the proportion of the derived cluster sizes in the permutation distribution that were larger than the observed group difference.

\section{Follow-up Analyses of the Identified Functional Network}

Following the strategy used in our prior work (Cao et al, 2016), we performed several supplementary analyses to probe the validity of the identified subnetwork by examining its test-retest reliability, neuropsychological associations, and potential structural confounds. Here, we reduced data dimensionality by averaging the connectivity of subnetwork links and gray matter volumes of subnetwork nodes for each subject. This aimed to increase sensitivity and to stay consistent with the NBS method per se, which treats the whole cluster as an entity for which the null hypothesis can only be rejected at the cluster level (Zalesky et al, 2010).

Test-retest reliability. Test-retest reliability is an important quality measure for a potential imaging phenotype. Here, we reanalyzed the test-retest data from our prior study (Cao et al, 2014) where 26 healthy subjects were scanned twice with the same emotional face-matching task within 2 consecutive weeks (mean time interval: $14.6 \pm 2.1$ days, mean age: $24.4 \pm 2.8$ years, 11 males, see (Cao et al, 2014) for sample details). Data processing and analysis followed the same procedures described above, and the mean connectivity estimates of the subnetwork were extracted for each subject and session. Intra-class correlation coefficients (ICCs) were used to quantify reliability, including $\operatorname{ICC}(2,1)$ and $\operatorname{ICC}(3,1)$, which reflect absolute agreement and relative consistency of the measurements between sessions, respectively. Based on established criteria (Cicchetti and Sparrow, 1981), ICC >0.40 indicates fair reliability, and ICC $>0.59$ indicates good reliability.

Relationship to emotion regulation. As 5-HTTLPR is a key regulator of the serotonin system and has been related to (mal)adaptive responses to emotional experiences (Caspi et al, 2003), we further examined whether individuals with higher or lower subnetwork connectivity differed in their preferred tendency to regulate emotions. Here, we analyzed the subscale scores of the ERQ, a 10-item scale measuring the respondents' tendency to regulate their emotions by either cognitive reappraisal or expressive suppression (Gross and John, 2003). For the analysis, we median-split participants into low and high connectivity groups and used ANCOVA models for the statistical comparison of ERQ subscale scores between groups, controlling for age, sex and site. Significance was set at $P<0.05$ after multiple corrections for both subscales.

Voxel-based morphometry (VBM). As 5-HTTLPR genotype has been associated with altered volume of several brain regions in earlier studies (Frodl et al, 2008; Pezawas et al, 2005), we further tested whether the identified functional alterations were related to gray matter differences. Here, we analyzed high-resolution T1 structural data for each subject with VBM (details see Supplementary Material). The mean gray matter volumes across all subnetwork nodes for each subject were entered as dependent variable into an ANCOVA model with genotype $(l l, l s, s s)$ as variable of interest and age, sex, and site as nuisance covariates. In addition, partial correlation was used to examine the association between mean gray matter volume and mean subnetwork connectivity while controlling for age, sex, and site.

Tract-based spatial statistics (TBSS). Earlier studies suggest an association between 5-HTTLPR genotype and microstructural integrity of brain white matter tracts (Benedetti et al, 2015; Pacheco et al, 2009). To test potential white matter correlates, we performed an analysis for participants with available DTI data (141 subjects, $39 l l, 62$ $l s, 40 \mathrm{ss})$ using TBSS implemented in the FMRIB Diffusion Toolbox (http://www.fmrib.ox.ac.uk/fsl/, see Supplementary Materials for further details). In addition, given that only 141 individuals had available DTI data, we further added the DTI data of additional 89 healthy subjects from a different cohort into the analysis to achieve a comparable sample size between the functional and structural studies. The added subjects were recruited from communities in and around Mannheim (mean age $26.52 \pm 9.28$ years, 35 males). The same DTI analysis was then performed in the combined sample (in total 230 subjects, $65 l l, 106 l s, 59 s s$ ).

\section{RESULTS}

\section{Amygdala Activation Analysis}

In our sample of 223 healthy subjects, we did not detect significant differences in amygdala activation between the three genotypes, neither in the regression (small-volume $P_{F W E}=0.80$ ) nor in the ANCOVA model (small-volume $\left.P_{F W E}=0.79\right)$.

\section{Network-Based Functional Connectivity Analysis}

The NBS analysis identified a functional cluster showing significantly negative correlation between the connectivity estimates of the cluster links and the number of risk allele $s$ $\left(P_{F W E}=0.03\right.$, Figure 1a). The cluster consisted of 14 links interconnecting 15 pairs of nodes that primarily mapped to the limbic system (hippocampus, orbitofrontal cortex, anterior cingulate gyrus), visual system (middle occipital gyrus, fusiform gyrus, middle, and inferior temporal gyrus) and subcortex (putamen and thalamus) (see Table 2 for details). The mean connectivity across all links in the cluster was significantly decreased in $l s$ and $s s$ carriers $(P=7 \mathrm{e}-15$, Figure 1b).

\section{Test-Retest Reliability of the Phenotype}

Analysis of the test-retest data revealed relatively high ICC values for the connectivity metrics of the identified subnetwork $(\operatorname{ICC}(2,1)=0.69, \operatorname{ICC}(3,1)=0.68)$. This suggests good 
a
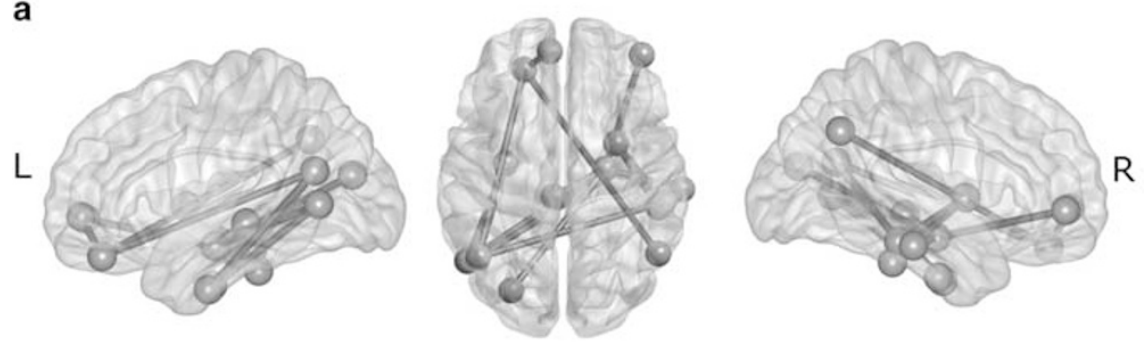

C

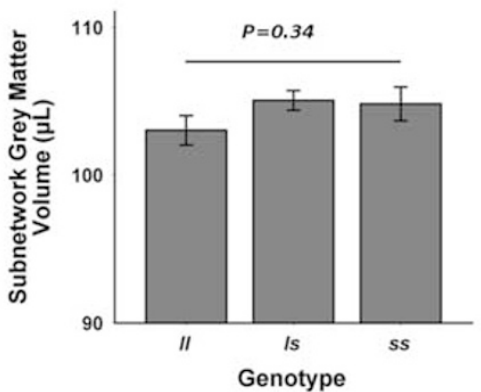

d

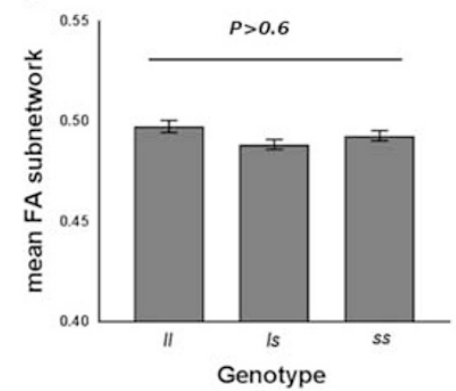

b

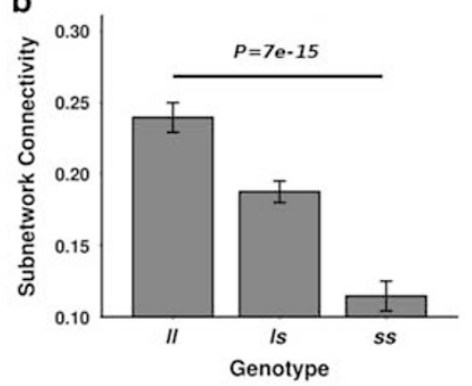

e

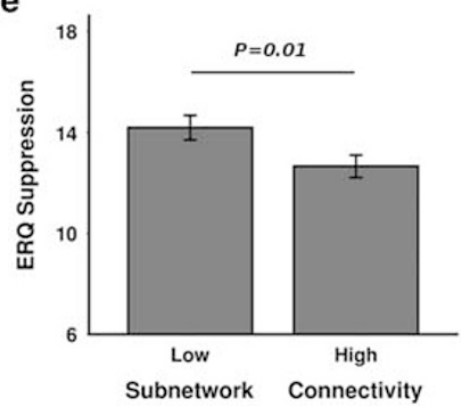

Figure I The identified subnetwork associated with 5-HTTLPR polymorphism (a). The functional connectivity of this subnetwork significantly decreased in both Is and ss carriers (cluster-level $P_{F W E}=0.03$, mean connectivity differences $\left.P=7 e-15\right)(b)$. In contrast, the mean grey matter volumes and mean FA values were not significantly different between genotype groups for the identified nodes (c) and interconnecting visual-limbic white matter tracts (d), respectively. In addition, participants with lower subnetwork connectivity showed higher emotional suppression scores (e). Bars indicate mean values and error bars indicate standard errors.

Table 2 Details for Nodes and Links in the Identified Subnetwork Ranked by Initial P-values

\begin{tabular}{|c|c|c|c|}
\hline & Links & & Initial uncorrected $P$-values \\
\hline । & L. thalamus ${ }^{\mathrm{a}}$ & R. hippocampus ${ }^{\mathrm{a}}$ & $4 e-6$ \\
\hline 2 & L. middle temporal gyrus ${ }^{b}$ & L. orbitofrontal cortex ${ }^{\mathrm{b}}$ & $5 e-6$ \\
\hline 4 & L. middle temporal gyrus ${ }^{b}$ & R. hippocampus ${ }^{\mathrm{a}}$ & $\mid e-5$ \\
\hline 5 & L. anterior cingulate cortex ${ }^{a}$ & L. orbitofrontal cortex ${ }^{\mathrm{b}}$ & $2 e-5$ \\
\hline 8 & R. angular gyrus ${ }^{\mathrm{b}}$ & L. orbitofrontal cortex ${ }^{\mathrm{b}}$ & $4 e-5$ \\
\hline 9 & L. middle temporal gyrus ${ }^{b}$ & R. fusiform gyrus ${ }^{\mathrm{b}}$ & $5 e-5$ \\
\hline 10 & L. middle occipital gyrus ${ }^{c}$ & R. hippocampus ${ }^{a}$ & $6 e-5$ \\
\hline 11 & L. fusiform gyrus ${ }^{b}$ & L. middle temporal gyrus ${ }^{b}$ & $7 e-5$ \\
\hline 12 & R. inferior temporal gyrus ${ }^{c}$ & L. middle temporal gyrus ${ }^{\mathrm{b}}$ & $7 e-5$ \\
\hline
\end{tabular}

Regions with 5-HTT expression in postmortem human brain (Kish et al, 2005; Varnas et al, 2004).

${ }^{b}$ Regions with 5-HTT expression in humans as implicated by positron emission tomography (Savli et al, 20I2).

${ }^{\mathrm{N}}$ No data on $5-\mathrm{HTT}$ expression available in humans.

reliability of the NBS-based subnetwork connectivity estimates.

\section{Relationship to Emotion Regulation}

The ANCOVA model revealed a significant difference between the low and high subnetwork connectivity groups with respect to the ERQ suppression scores $(F=6.71$,
$P=0.01$, Figure 1e). Here, the group with the lower connectivity showed significantly higher emotion suppression scores than that with the higher connectivity, suggesting that healthy individuals with a lower coupling of the subnetwork are more likely to employ a maladaptive strategy for emotion regulation. No significant group difference was detected for the ERQ reappraisal scores $(F=0.78, P=0.38)$. 


\section{Specificity of the Identified Genetic Association}

In a supplementary analysis we further investigated the specificity of the identified genetic association. For this, we randomly selected 10000 SNPs from the human genome and tested them for potential associations with mean connectivity estimates of the identified subnetwork (inclusion criterion: at least 20 individuals in each genotype subgroup, not in significant linkage disequilibrium with 5-HTTLPR as defined by $\left.R^{2}<0.8\right)$. Notably, no significant association was observed for any of these randomly selected SNPs after Bonferroni correction for multiple comparisons. In contrast, the association of 5-HTTLPR with subnetwork connectivity remained significant after correction for multiple comparisons of all tested 10000 SNPs (see Supplementary Figure S1 and Supplementary Materials for further information). These data argue for the genetic specificity of our main finding and against a false positive result.

\section{Gray Matter Structural Analysis}

The VBM analysis revealed no significant differences in mean gray matter volumes of the subnetwork nodes between genotype groups $(F=1.08, P=0.34$, Figure $1 \mathrm{c})$ and no significant correlation between mean gray matter volumes and subnetwork connectivity measures $(r=0.05, P=0.45)$. This suggests that the identified functional network alteration is independent of genotype-related differences in gray matter.

\section{White Matter Structural Analysis}

The initial TBSS analysis of 141 individuals revealed no significant differences in FA between genotype groups in visual-limbic fiber bundles $\left(P_{F W E}>0.6\right.$, Figure 1d). The inclusion of 89 additional subjects further confirmed this negative finding $\left(P_{F W E}>0.62\right)$. Extending the FA analysis to the whole brain white matter skeleton did not reveal any genotype effect either $\left(P_{F W E}>0.27\right)$. Similar results emerged in additional analyses focusing on radial and axial diffusivity $\left(P_{F W E}>0.22\right)$. Together these findings suggest that the identified functional network alteration is independent of genotype-related differences in white matter structure.

\section{DISCUSSION}

This study used multimodal neuroimaging to identify a potential functional connectivity phenotype associated with the 5-HTTLPR genotype at the brain system level. Our analyses yielded several findings, which we will discuss in the following.

First, in line with recent reports (Bastiaansen et al, 2014; Murphy et al, 2013; Viviani et al, 2010), we failed to replicate the association between 5-HTTLPR variation and amygdala activation described in earlier studies (Hariri et al, 2005; 2002). The negative finding in our relatively large sample supports the conclusions of recent meta-analyses, which also failed to find a convincing association and highlighted a strong publication bias towards a large number of small and underpowered neuroimaging studies with positive findings (Bastiaansen et al, 2014; Murphy et al, 2013). This outcome is not too surprising given that the amygdala strongly habituates to the recurrent presentations of emotional stimuli and yields unreliable activation estimates (Johnstone et al, 2005; Plichta et al, 2012). Another plausible contributor to earlier positive findings is the wealth of other genetic (Pezawas et al, 2008; Surguladze et al, 2012) and environmental factors (Rabl et al, 2014; van der Doelen et al, 2014) that potentially interact with 5-HTTLPR, making underpowered studies more vulnerable to hidden bias (Bastiaansen et al, 2014). Finally, recent work has identified a strong citation preference for 5-HTTLPR studies with positive outcomes and/or optimistic connotations, making a systematic distortion of the related evidence base probable (de Vries et al, 2016). Together, these findings and our data encourage the critical re-evaluation of amygdala activation as putative neural mechanism linking 5-HTTLPR to emotional behavior and the vulnerability to psychopathology.

Second, we detected a significant effect of 5-HTTLPR on the functional connectivity of a visual-limbic subnetwork. Here, the $s$ allele was associated with a dose-dependent decrease in the coupling of several brain regions involved in emotion perception and regulation, including fusiform gyrus, anterior cingulate gyrus, hippocampus, orbitofrontal cortex, striatum, and thalamus (Etkin et al, 2015; Phillips et al, 2003). Among others, the anterior cingulate cortex is a crucial hub for top-down control of limbic regions (Etkin et al, 2015), the orbitofrontal cortex is associated with emotional decision making (Bechara et al, 2000), and the hippocampus is a key structure for emotion-related memory processing (Etkin et al, 2015; Phillips et al, 2003). The conjoint involvement of this network in emotion processing makes it a plausible target for 5-HTTLPR genetic variation.

Notably, a relative enrichment of 5-HTT (Kish et al, 2005; Varnas et al, 2004) and serotonin receptors (Biegon et al, 1986; Varnas et al, 2004) has been demonstrated for several subnetwork regions in humans (see Table 2). In addition, animal studies point to a sensitivity of these regions to 5HTTLPR variation, where the 5-HTT gene knock-out mice are related to enhanced extracellular serotonin level and reduced serotonin receptor gene expression in the hippocampus (Fabre et al, 2000; Mathews et al, 2004), striatum (Mathews et al, 2004; Rioux et al, 1999), and prefrontal cortex (Mathews et al, 2004). In humans, the $s$ allele relates to significantly lower 5-HTT binding potentials in the striatum (Praschak-Rieder et al, 2007) and 5-HT1A receptor binding potentials in the hippocampus, anterior cingulate cortex, orbitofrontal cortex, and lateral temporal lobe (David et al, 2005). Moreover, altered functional connectivity of part of the identified subnetwork has previously been found in $s$ allele carriers, in particular the anterior cingulate cortex (Pezawas et al, 2005), prefrontal cortex (Heinz et al, 2005), and fusiform gyrus (Kruschwitz et al, 2015). Overall, these findings and our data support the idea that the neural mechanism of 5-HTTLPR involves a large-scale network of conjointly interacting visual-limbic brain regions.

Third, we conducted several follow-up analyses to probe the robustness of the identified subnetwork, its relationship to emotion regulation, and potential structural basis. Consistent with our previous work (Cao et al, 2014), we demonstrated good test-retest reliability of the subnetwork metrics. This outcome is in stark contrast to the poor robustness of amygdala activation measures (Plichta et al, 2012) and encourages the use of network-based approaches 
in search for functional neuroimaging phenotypes in emotional systems.

We further explored the relationship between the identified subnetwork and two important emotion regulation strategies, namely cognitive reappraisal and behavioral suppression (Gross and John, 2003). Cognitive reappraisal is an adaptive emotion regulation strategy that reduces the negative salience and somatic consequences of emotional experiences by cognitive reframing. In contrast, the emotion suppression strategy is considered maladaptive, as it merely inhibits the behavioral expression of emotional experiences without effectively reducing negative emotions and their stress-related somatic consequences (Gross, 2002; Gross and John, 2003). Here, we observed significantly higher suppression scores in the lower subnetwork connectivity group, suggesting a stronger tendency to inhibit behavioral responses to emotional experiences. Notably, it has been demonstrated that emotion suppression relates to increased stress response to negative events (Gross, 2002), bringing detrimental effects on a variety of health-protective variables including well-being, positive emotions, social functioning, social support, and memory (Gross, 2002; Gross and John, 2003). As a consequence, individuals with a stronger tendency to suppress their emotions are at increased risk for stress-related psychiatric conditions such as mood and anxiety disorders (Gross, 2002). Our findings complement these data and suggest that the neural mechanism linking 5-HTTLPR to emotional regulation and risk for psychopathology includes connectivity deficits in the visual-limbic system.

We also examined the potential structural basis for our results. Here, the VBM and TBSS analyses provided no evidence for associations between 5-HTTLPR and structural measures, suggesting that the identified network phenotype is primarily functional and unlikely related to hidden structural alterations.

Several limitations of our study are worth considering. First, although the present work examined a relatively large group of individuals, our findings merit further replication in larger cohorts. Second, although we balanced our genotype groups for a broad range of demographic, psychological, task performance, and data quality-related variables, we cannot fully exclude that our findings were not influenced by confounds that we may have missed. Third, despite the increasing popularity of NBS-based approaches in the recent literature, the identified connectivity difference can only be interpreted at the cluster level. Fourth, future studies are advised to explore the significance of the identified subnetwork in clinical populations, in particular patients with anxiety disorders and depression.

In conclusion, our data provide evidence for coupling deficits in the visual-limbic system as a network-based neuroimaging phenotype of 5-HTTLPR variation. The identified phenotype is test-retest reliable, relates to a maladaptive emotion regulation strategy, and is independent of structural alterations of the identified network nodes and links. These findings encourage the study of visual-limbic network deficits in larger cohorts and clinical populations, and suggest that further critical evaluations of the association between 5-HTTLPR genotype and amygdala activation are warranted.

\section{FUNDING AND DISCLOSURE}

AM-L has received consultant fees from AstraZeneca, Elsevier, F Hoffmann-La Roche, Gerson Lehrman Group, Lundbeck, Outcome Europe Sárl, Outcome Sciences, Roche Pharma, Servier International, and Thieme Verlag, and has received lecture fees including travel expenses from Abbott, AstraZeneca, Aula Médica Congresos, BASF, Boehringer Ingelheim, Groupo Ferrer International, Janssen-Cilag, Lilly Deutschland, LVR Klinikum Düsseldorf, Otsuka Pharmaceuticals, and Servier Deutschland. The remaining authors declare no conflicts of interest.

\section{ACKNOWLEDGMENTS}

This study is supported by the German Federal Ministry of Education and Research (IntegraMent: grants 01ZX1314A, 01ZX1314B and 01ZX1314G to MMN, SC, MR, AM-L, HW, and AH; NGFNplus MooDS: grants 01GS08144, 01GS08147, 01GS08148 to MMN, SC, HW, MR, AM-L and AH), the European Community's Seventh Framework Programme under the grant agreements No. 115300 (Project EU-AIMS to AM-L), No 115008 (Project EU-NEWMEDS to AM-L), No. 602805 (Project EU-AGGRESSOTYPE to AM-L) and No. 602450 (Project EU-IMAGEMEND to AM-L), and the German Research Foundation (DFG; grant FOR2107; RI908/11-1 to MR; WI3429/3-1 to SHW). HT acknowledges grant support by the German Federal Ministry of Education and Research (grant 01GQ1102). We thank Leila Haddad, Oliver Grimm, Christine Esslinger, Dagmar Gass, Daniela Mier and Carina Sauer for research assistance.

\section{REFERENCES}

Bastiaansen JA, Servaas MN, Marsman JB, Ormel J, Nolte IM, Riese $\mathrm{H}$ et al (2014). Filling the gap: relationship between the serotonin-transporter-linked polymorphic region and amygdala activation. Psychol Sci 25: 2058-2066.

Bechara A, Damasio H, Damasio AR (2000). Emotion, decision making and the orbitofrontal cortex. Cereb Cortex 10: 295-307.

Benedetti F, Bollettini I, Poletti S, Locatelli C, Lorenzi C, Pirovano A et al (2015). White matter microstructure in bipolar disorder is influenced by the serotonin transporter gene polymorphism 5-HTTLPR. Genes Brain Behav 14: 238-250.

Biegon A, Kargman S, Snyder L, McEwen BS (1986). Characterization and localization of serotonin receptors in human brain postmortem. Brain Res 363: 91-98.

Budisavljevic S, Kawadler JM, Dell'Acqua F, Rijsdijk FV, Kane F, Picchioni M et al (2016). Heritability of the limbic networks. Soc Cogn Affect Neurosci 11: 746-757.

Canli T, Lesch KP (2007). Long story short: the serotonin transporter in emotion regulation and social cognition. Nat Neurosci 10: 1103-1109.

Cao H, Bertolino A, Walter H, Schneider M, Schafer A, Taurisano P et al (2016). Altered functional subnetwork during emotional face processing: a potential intermediate phenotype for schizophrenia. JAMA Psychiatry 73: 598-605.

Cao H, Plichta MM, Schafer A, Haddad L, Grimm O, Schneider M et al (2014). Test-retest reliability of fMRI-based graph theoretical properties during working memory, emotion processing, and resting state. Neuroimage 84: 888-900. 
Caspi A, Sugden K, Moffitt TE, Taylor A, Craig IW, Harrington H et al (2003). Influence of life stress on depression: moderation by a polymorphism in the 5-HTT gene. Science 301: 386-389.

Cicchetti DV, Sparrow SA (1981). Developing criteria for establishing interrater reliability of specific items: applications to assessment of adaptive behavior. Am J Ment Defic 86: 127-137.

David SP, Murthy NV, Rabiner EA, Munafo MR, Johnstone EC, Jacob $\mathrm{R}$ et al (2005). A functional genetic variation of the serotonin (5-HT) transporter affects 5-HT1A receptor binding in humans. J Neurosci 25: 2586-2590.

de Vries YA, Roest AM, Franzen M, Munafo MR, Bastiaansen JA (2016). Citation bias and selective focus on positive findings in the literature on the serotonin transporter gene (5-HTTLPR), life stress and depression. Psychol Med 46: 2971-2979.

Dima D, Roberts RE, Frangou S (2016). Connectomic markers of disease expression, genetic risk and resilience in bipolar disorder. Transl Psychiatry 6: e706.

Etkin A, Buchel C, Gross JJ (2015). The neural bases of emotion regulation. Nat Rev Neurosci 16: 693-700.

Fabre V, Beaufour C, Evrard A, Rioux A, Hanoun N, Lesch KP et al (2000). Altered expression and functions of serotonin 5-HT1A and 5-HT1B receptors in knock-out mice lacking the 5-HT transporter. Eur J Neurosci 12: 2299-2310.

Frodl T, Koutsouleris N, Bottlender R, Born C, Jager M, Morgenthaler $\mathrm{M}$ et al (2008). Reduced gray matter brain volumes are associated with variants of the serotonin transporter gene in major depression. Mol Psychiatry 13: 1093-1101.

Gross JJ (2002). Emotion regulation: affective, cognitive, and social consequences. Psychophysiology 39: 281-291.

Gross JJ, John OP (2003). Individual differences in two emotion regulation processes: implications for affect, relationships, and well-being. J Pers Soc Psychol 85: 348-362.

Hariri AR, Drabant EM, Munoz KE, Kolachana BS, Mattay VS, Egan MF et al (2005). A susceptibility gene for affective disorders and the response of the human amygdala. Arch Gen Psychiatry 62: 146-152.

Hariri AR, Holmes A (2006). Genetics of emotional regulation: the role of the serotonin transporter in neural function. Trends Cogn Sci 10: 182-191.

Hariri AR, Mattay VS, Tessitore A, Kolachana B, Fera F, Goldman D et al (2002). Serotonin transporter genetic variation and the response of the human amygdala. Science 297: 400-403.

Heinz A, Braus DF, Smolka MN, Wrase J, Puls I, Hermann D et al (2005). Amygdala-prefrontal coupling depends on a genetic variation of the serotonin transporter. Nat Neurosci 8: 20-21.

Hu X, Oroszi G, Chun J, Smith TL, Goldman D, Schuckit MA (2005). An expanded evaluation of the relationship of four alleles to the level of response to alcohol and the alcoholism risk. Alcohol Clin Exp Res 29: 8-16.

Johnstone T, Somerville LH, Alexander AL, Oakes TR, Davidson RJ, Kalin NH et al (2005). Stability of amygdala BOLD response to fearful faces over multiple scan sessions. Neuroimage 25: 1112-1123.

Kish SJ, Furukawa Y, Chang LJ, Tong J, Ginovart N, Wilson A et al (2005). Regional distribution of serotonin transporter protein in postmortem human brain: is the cerebellum a SERT-free brain region? Nucl Med Biol 32: 123-128.

Kruschwitz JD, Walter M, Varikuti D, Jensen J, Plichta MM, Haddad L et al (2015). 5-HTTLPR/rs25531 polymorphism and neuroticism are linked by resting state functional connectivity of amygdala and fusiform gyrus. Brain Struct Funct 220: 2373-2385.

Lesch KP, Bengel D, Heils A, Sabol SZ, Greenberg BD, Petri S et al (1996). Association of anxiety-related traits with a polymorphism in the serotonin transporter gene regulatory region. Science 274: $1527-1531$.

Liu X, Hairston J, Schrier M, Fan J (2011). Common and distinct networks underlying reward valence and processing stages: a meta-analysis of functional neuroimaging studies. Neurosci Biobehav Rev 35: 1219-1236.

Mathews TA, Fedele DE, Coppelli FM, Avila AM, Murphy DL, Andrews AM (2004). Gene dose-dependent alterations in extraneuronal serotonin but not dopamine in mice with reduced serotonin transporter expression. J Neurosci Methods 140: 169-181.

Murphy SE, Norbury R, Godlewska BR, Cowen PJ, Mannie ZM, Harmer CJ et al (2013). The effect of the serotonin transporter polymorphism (5-HTTLPR) on amygdala function: a metaanalysis. Mol Psychiatry 18: 512-520.

Pacheco J, Beevers CG, Benavides C, McGeary J, Stice E, Schnyer DM (2009). Frontal-limbic white matter pathway associations with the serotonin transporter gene promoter region (5-HTTLPR) polymorphism. J Neurosci 29: 6229-6233.

Pezawas L, Meyer-Lindenberg A, Drabant EM, Verchinski BA, Munoz KE, Kolachana BS et al (2005). 5-HTTLPR polymorphism impacts human cingulate-amygdala interactions: a genetic susceptibility mechanism for depression. Nat Neurosci 8: 828-834.

Pezawas L, Meyer-Lindenberg A, Goldman AL, Verchinski BA, Chen G, Kolachana BS et al (2008). Evidence of biologic epistasis between BDNF and SLC6A4 and implications for depression. Mol Psychiatry 13: 709-716.

Phillips ML, Drevets WC, Rauch SL, Lane R (2003). Neurobiology of emotion perception I: the neural basis of normal emotion perception. Biol Psychiatry 54: 504-514.

Plichta MM, Schwarz AJ, Grimm O, Morgen K, Mier D, Haddad L et al (2012). Test-retest reliability of evoked BOLD signals from a cognitive-emotive fMRI test battery. Neuroimage 60: 1746-1758.

Power JD, Cohen AL, Nelson SM, Wig GS, Barnes KA, Church JA et al (2011). Functional network organization of the human brain. Neuron 72: 665-678.

Praschak-Rieder N, Kennedy J, Wilson AA, Hussey D, Boovariwala A, Willeit M et al (2007). Novel 5-HTTLPR allele associates with higher serotonin transporter binding in putamen: a [(11)C] DASB positron emission tomography study. Biol Psychiatry 62: 327-331.

Rabl U, Meyer BM, Diers K, Bartova L, Berger A, Mandorfer D et al (2014). Additive gene-environment effects on hippocampal structure in healthy humans. J Neurosci 34: 9917-9926.

Rioux A, Fabre V, Lesch KP, Moessner R, Murphy DL, Lanfumey L et al (1999). Adaptive changes of serotonin 5-HT2A receptors in mice lacking the serotonin transporter. Neurosci Lett 262: 113-116.

Sabatinelli D, Fortune EE, Li Q, Siddiqui A, Krafft C, Oliver WT et al (2011). Emotional perception: meta-analyses of face and natural scene processing. Neuroimage 54: 2524-2533.

Savli M, Bauer A, Mitterhauser M, Ding YS, Hahn A, Kroll T et al (2012). Normative database of the serotonergic system in healthy subjects using multi-tracer PET. Neuroimage 63: 447-459.

Spreng RN, Mar RA, Kim AS (2009). The common neural basis of autobiographical memory, prospection, navigation, theory of mind, and the default mode: a quantitative meta-analysis. J Cogn Neurosci 21: 489-510.

Surguladze SA, Radua J, El-Hage W, Gohier B, Sato JR, Kronhaus DM et al (2012). Interaction of catechol O-methyltransferase and serotonin transporter genes modulates effective connectivity in a facial emotion-processing circuitry. Transl Psychiatry 2: e70.

Tzourio-Mazoyer N, Landeau B, Papathanassiou D, Crivello F, Etard O, Delcroix N et al (2002). Automated anatomical labeling of activations in SPM using a macroscopic anatomical parcellation of the MNI MRI single-subject brain. Neuroimage 15: 273-289.

van der Doelen RH, Deschamps W, D'Annibale C, Peeters D, Wevers RA, Zelena D et al (2014). Early life adversity and serotonin transporter gene variation interact at the level of the 
adrenal gland to affect the adult hypothalamo-pituitaryadrenal axis. Transl Psychiatry 4: e409.

Varnas K, Halldin C, Hall H (2004). Autoradiographic distribution of serotonin transporters and receptor subtypes in human brain. Hum Brain Mapp 22: 246-260.
Viviani R, Sim EJ, Lo H, Beschoner P, Osterfeld N, Maier C et al (2010). Baseline brain perfusion and the serotonin transporter promoter polymorphism. Biol Psychiatry 67: 317-322.

Zalesky A, Fornito A, Bullmore ET (2010). Network-based statistic: identifying differences in brain networks. Neuroimage 53: 1197-1207.

Supplementary Information accompanies the paper on the Neuropsychopharmacology website (http://www.nature.com/npp) 